\title{
Research Article \\ On the Localization of the Riesz Means of Multiple Fourier Series of Distributions
}

\author{
A. A. Rakhimov \\ Institute for Mathematical Research, Universiti Putra Malaysia, Serdang, 43300 Selangor, Malaysia \\ Correspondence should be addressed to A. A. Rakhimov, abdumalik2004@mail.ru \\ Received 1 August 2011; Accepted 13 September 2011 \\ Academic Editor: Gerd Teschke \\ Copyright (C) 2011 A. A. Rakhimov. This is an open access article distributed under the Creative \\ Commons Attribution License, which permits unrestricted use, distribution, and reproduction in \\ any medium, provided the original work is properly cited. \\ We study special partial sums of multiple Fourier series of distributions. We obtain sufficient con- \\ ditions of summation of Riesz means of Fourier expansions of distributions with compact sup- \\ port.
}

\section{Introduction}

Reconstruction of a distribution from its Fourier expansions is one of the recently studied problems in harmonic analysis. Well-defined sequence of partial sums always converges in the weak topology $[1,2]$. However, one can study these expansions in classical sense in the domains where a distribution coincides with locally integrable function. From the divergence of Fourier series of the Dirac delta function, it follows that a singularity of the distribution makes significant influence for the convergence in a domain where it is very smooth (equal to zero in this case). For eigenfunction expansions of even piecewise smooth functions, its discontinuity has negative effect on convergence at the point far from singularity sets (Pinsky phenomenon) [3-5].

In the present paper, we consider a localization problem of multiple Fourier series of distributions. Unlike one-dimensional case partial sums of multiple Fourier series can be defined in various ways, such as rectangular, square, and spherical partial sums [1,2]. Fourier expansions of a singular distribution can be also studied in the classical sense in the domains where it coincides with a regular function $[1,2,6-13]$. We prove a localization theorem for nonspherical partial sums, that is, for Fourier series under summation over domains bounded by level surfaces of elliptic polynomials. 


\section{Preliminaries and Formulation of the Main Result}

We denote $C^{\infty}\left(T^{N}\right)$-the space of $2 \pi$-periodic in each variable, infinitely differentiable on $R^{N}$ functions, where $T^{N}=\left\{x \in R^{N}:-\pi<x_{j} \leq \pi\right\}$.

Let $\gamma=\left(\gamma_{1}, \gamma_{2}, \ldots, \gamma_{N}\right)$ denote a multiindex, that is, $N$-dimensional vector with integer nonnegative components and let $D^{\gamma}=D_{1}^{\gamma_{1}} D_{2}^{\gamma_{2}} \cdots D_{N}^{\gamma_{N}}$, where $D_{j}=(1 / i)\left(\partial / \partial x_{j}\right)$.

The system of seminorms $\operatorname{Sup}_{x \in T^{N}}\left|D^{\gamma} f(x)\right|$ produces a locally convex topology in $C^{\infty}\left(T^{N}\right)$, where $\gamma$ runs over all multiindexes. We denote $\varepsilon\left(T^{N}\right)$ corresponding locally convex topological space. Let $\varepsilon^{\prime}\left(T^{N}\right)$ be the space of periodic distributions, that is, the space of continuous linear functionals on $\varepsilon\left(T^{N}\right)$.

For any distribution $f$ from $\varepsilon^{\prime}\left(T^{N}\right)$ we define its Fourier coefficients $f_{n}$ as the action of distribution $f$ on the test function $(2 \pi)^{-N / 2} \exp (-i n x)$, where $x \in T^{N}$ and $n \in Z^{N}, N$-dimensional vector with integer coordinates. Then $f$ can be represented by Fourier series

$$
f=(2 \pi)^{-N / 2} \sum_{n \in Z^{N}} f_{n} \exp (i n x),
$$

which always converges in the weak topology (see, e.g., in [1, 2]).

Consider the following polynomial:

$$
P_{m}(n)=\left(\sum_{j=1}^{r+1} n_{j}^{2}\right)^{m+1}+\left(\sum_{j=r+2}^{N} n_{j}^{2}\right)^{m}\left(\sum_{j=1}^{N} n_{j}^{2}\right),
$$

where $n=\left(n_{1}, n_{2}, \ldots, n_{N}\right) \in Z^{N}, m$ is a positive integer number, and $r=0,1, \ldots, N-1$.

Polynomial $P_{m}(n)$ is a homogeneous of degree $2(m+1)$, that is,

$$
P_{m}(\lambda \cdot n)=\lambda^{2(m+1)} \cdot P_{m}(n),
$$

and an elliptic, that is,

$$
P_{m}(n)>0, \quad n \neq 0 .
$$

Thus, a family of bounded sets

$$
\Lambda(\lambda)=\left\{n \in Z^{N}: P_{m}(n)<\lambda\right\}, \quad \lambda \in R^{+},
$$

enjoying the following properties:

(a) for any pairs $\left(\lambda_{1}, \lambda_{2}\right) \in R^{+} \times R^{+}$, there is $\lambda \in R^{+}$, such that $\Lambda\left(\lambda_{1}\right) \cup \Lambda\left(\lambda_{2}\right) \subset \Lambda(\lambda)$,

(b) $\bigcup_{\lambda \in R^{+}} \Lambda(\lambda)=Z^{N}$.

Let $f \in \varepsilon^{\prime}\left(T^{N}\right)$. Then $\Lambda$-partial sums of series (2.1) define by equality

$$
E_{\lambda} f(x)=(2 \pi)^{-N / 2} \sum_{\Lambda(\lambda)} f_{n} \exp (\text { in } \cdot x) .
$$


For any real $s, s \geq 0$, we define the Riesz means of (2.6) by

$$
E_{\lambda}^{S} f(x)=(2 \pi)^{-N / 2} \sum_{\Lambda(\lambda)}\left(1-\frac{P_{m}(n)}{\lambda}\right)^{s} f_{n} \exp (i n x) .
$$

At $s=0$ we obtain partial sums (2.6).

Summability of series (2.1), as well as its regularization (2.7), depends on power of singularity of $f$. In order to classify singularities of distributions, we apply periodic Liouville spaces $L_{p}^{\alpha}\left(T^{N}\right), 1<p \leq \infty, \alpha \in R[14]$.

In this paper we study the convergence of the Riesz means (2.7) in domains where a distribution coincides with the zero (the localization problem). The main result of this work is to prove the following.

Theorem 2.1. Let $f \in L_{p}^{-\alpha}\left(T^{N}\right) \cap \varepsilon^{\prime}\left(T^{N}\right), 1<p \leq 2, \alpha>0$, and coincide with zero in $\Omega \subset T^{N}$. If

$$
s>\max \left\{\frac{(N-r-1)(1-1 / 2 m)}{p}+\frac{r}{2}, \frac{N-1}{2}\right\}+\alpha,
$$

then uniformly on any compact set $K \subset \Omega$

$$
\lim _{\lambda \rightarrow \infty} E_{\lambda}^{S} f(x)=0
$$

\section{Auxiliary Lemmas on Estimations of the Dirichlet Kernel}

The Riesz means (2.7) can be written as

$$
E_{\lambda}^{s} f(x)=\left\langle f, D_{\lambda}^{s}(x-y)\right\rangle
$$

where $f$ acts on $D_{\lambda}^{s}(x-y)$ by $y$ and $D_{\lambda}^{s}(x)$ is the Riesz means of $\Lambda$-partial sums of multiple Fourier series of the Dirac Delta Function:

$$
D_{\lambda}^{s}(x)=(2 \pi)^{-N / 2} \sum_{\Lambda(\lambda)}\left(1-\frac{P_{m}(n)}{\lambda}\right)^{s} \exp (\text { inx } x) .
$$

Note that, if $r=N-1$, then $D_{\lambda}^{s}(x)$ is exactly the Riesz means of the Dirichlet Kernel.

First, we estimate (3.2) in the norm of positive Liouville spaces. In this, we use the relation between the kernel (3.2) and the relevant kernel of Fourier integrals. Such a relation is known as the Poisson Summation Formula. The kernel for the corresponding Fourier integrals can be also described by the same polynomial $P_{m}$ replacing its argument range from $n \in Z^{N}$ to $\xi \in R^{N}$ :

$$
\Theta_{\lambda}^{s}(x)=(2 \pi)^{-N / 2} \int_{\Lambda(\lambda)}\left(1-\frac{P_{m}(\xi)}{\lambda}\right)^{s} \exp (i \xi \cdot x) d \xi
$$

where in definition of the domain $\Lambda(\lambda)$ its range must be changed accordantly. 
Following asymptotic formula valid for the kernel (3.3), we obtain Lemma 3.1.

Lemma 3.1. Let $x \in R^{N}, x=\left(x^{\prime}, x^{\prime \prime}\right), x^{\prime} \in R^{r+1}, x^{\prime \prime} \in R^{N-r-1}, 0<\delta_{0}<\left|x^{\prime}\right|, \mu=\lambda^{1 / 2(m+1)}$. Then, for $\left|x^{\prime \prime}\right|<\varepsilon \mu^{-(1-1 / 2 m)}, 0<\varepsilon<1 / 2$, and $\mu \rightarrow \infty$

$$
\Theta_{\lambda}^{s}(x)=\frac{c \mu^{N} \cos \left(\mu\left|x^{\prime}\right|+(r / 2-s)(\pi / 2)\right)}{\left(\mu\left|x^{\prime}\right|\right)^{((r+2) / 2)+s+((N-1-r) / 2 m)}} \times\left(1+O\left(\frac{1}{\mu|x|}\right)+O\left(\left|x^{\prime \prime}\right| \mu^{1-1 / 2 m}\right)\right) .
$$

Note, that, integral operators, corresponding to the kernels $D_{\lambda}^{s}$ and $\Theta_{\lambda}^{s}$, act in different functional spaces. On the assumptions of Lemma 3.1 it follows that, if $s$ satisfies condition (2.8) in Theorem 2.1, then $\Theta_{\lambda}^{s} \in L_{1}$.

Suppose that $f \in L_{1}\left(T^{N}\right)$ vanishes near the boundary of the cube $T^{N}$. Then the function

$$
g(x)= \begin{cases}f(x), & \text { if } x \in T^{N} \\ 0, & \text { if } x \notin T^{N}\end{cases}
$$

belongs to $L_{1}\left(R^{N}\right)$ and preserves all properties of $f$ in the interior of $T^{N}$. Conversely, if $g \in$ $L\left(R^{N}\right)$ has its support in the interior of $T^{N}$, then, if we shift its graph along the coordinate axes with steps which are multiples of $2 \pi$, we get a periodic function $f \in L_{1}\left(T^{N}\right)$, which coincides with $g$ on $T^{N}$, that is,

$$
f(x)=\sum_{n \in Z^{N}} g(x+2 \pi n) .
$$

Fourier coefficient of $f \in T^{N}$ and the Fourier transformation of $g \in L_{1}\left(R^{N}\right)$ are related by the formula $f_{n}=(2 \pi)^{-N / 2} \widehat{g}(n)$. Thus, comparing this with previous formula, we obtain the Poisson summation formula

$$
\sum_{n \in Z^{N}} g(x+2 \pi n)=(2 \pi)^{-N / 2} \sum_{n \in Z^{N}} \widehat{g}(n) \exp i n x .
$$

The Poisson summation formula (3.7) holds, for example, if the function $g$ satisfies conditions

$$
|g(x)| \leq(1+|x|)^{-N-\epsilon}, \quad|\widehat{g}(\xi)| \leq(1+|\xi|)^{-N-\epsilon}
$$

where $\epsilon$ is any positive number.

Note that from definition of the kernel $\Theta_{\lambda}^{s}$ it follows that

$$
\widehat{\Theta}_{\lambda}^{s}(n)= \begin{cases}(2 \pi)^{-N / 2}\left(1-\frac{P_{m}(n)}{\lambda}\right)^{s}, & \text { if } P_{m}(n) \leq \lambda, \\ 0, & \text { otherwise. }\end{cases}
$$

Equality (3.9) establishes relationships between the Fourier coefficients of the kernel $D_{\lambda}^{s}$ and the Fourier transformation of the kernel $\Theta_{\lambda}^{s}$. Moreover, from Lemma 3.1 and 
inequality (2.8), it follows that the kernel $\Theta_{\lambda}^{s}$ satisfies conditions (3.8). Thus, from (3.7), we obtain

$$
\sum_{n \in Z^{N}} \Theta_{\lambda}^{s}(x+2 \pi n)=(2 \pi)^{-N / 2} \sum_{n \in Z^{N}} \widehat{\Theta}_{\lambda}^{s}(n) \exp i n x
$$

On the other hand, taking into account (3.9), from the definition of the kernel $D_{\lambda}^{s}$, we obtain

$$
D_{\lambda}^{S}(x)=(2 \pi)^{-N / 2} \sum_{n \in Z^{N}} \widehat{\Theta}_{\lambda}^{S}(n) \exp i n x
$$

Thus, from (3.10) and (3.11), we obtain

$$
D_{\lambda}^{s}(x)=\sum_{n \in Z^{N}} \Theta_{\lambda}^{s}(x+2 \pi n)
$$

Then in (3.12) separating the term $n=0$ we obtain

$$
D_{\lambda}^{s}(x)=\Theta_{\lambda}^{s}(x)+\Theta_{*, \lambda}^{s}(x)
$$

where $\Theta_{*, \lambda}^{s}(x)$ is defined as

$$
\Theta_{*, \lambda}^{s}(x)=\sum_{n \in Z^{N}, n \neq 0} \Theta_{\lambda}^{s}(x+2 \pi n)
$$

Then from Lemma 3.1 we immediately obtain the following lemma.

Lemma 3.2. Let $\varepsilon>0$ be an arbitrary small number and $\left|x_{i}\right| \leq 2 \pi-\varepsilon$, for any $i=1,2,3, \ldots, N$. If $s$ satisfies (2.8), then

$$
\Theta_{*, \lambda}^{s}(x)=O\left(\lambda^{1 / 2(m+1)}\right)^{N-s-1-(r / 2)-((N-1-r) / 2 m)} .
$$

Lemma 3.2 provides an estimation of the second term in (3.13). Moreover, if $0<\delta_{0}<\left|x^{\prime}\right|$, then from (3.4) we obtain an estimation for the first term in (3.13). Thus, we proved the following lemma.

Lemma 3.3. Let $\varepsilon>0$ be an arbitrary small number and $\left|x^{\prime}\right|>\varepsilon$. If $s$ satisfies (2.8), then

$$
D_{\lambda}^{S}(x)=O\left(\lambda^{1 / 2(m+1)}\right)^{N-s-1-(r / 2)-((N-1-r) / 2 m)} .
$$

We will estimate the kernel $D_{\lambda}^{s}(x)$ in the norm of $L_{q}\left(T^{N}\right)$ space. Lemma 3.3 provides an estimation at $q=\infty$. If $q=2$, then we have the following estimation see [15]. 
Theorem 3.4. Let $K \subset \subset T^{N}$ be a compact set, then uniformly by $x \in K$

$$
\left\|D_{\lambda}^{s}(x-y)\right\|_{L_{2}(F)}=O\left(\lambda^{(N-1-2 s) / 4(m+1)}\right)
$$

where $F$ is an arbitrary domain in $T^{N}$ such that $\bar{F} \cap K=\emptyset$.

Then using Stein's interpolation theorem for analytical family of linear operators [16] with $q=\infty$ and $q=2$, we obtain the following.

Lemma 3.5. Let s satisfy (2.8), and let $K \subset \subset T^{N}$ be an arbitrary compact set. Then uniformly by $x \in K$

$$
\left\|D_{\lambda}^{S}(x-y)\right\|_{L_{q}(F)}=O\left(\lambda^{1 / 2(m+1)}\right)^{N-S-1-(r / 2)-((N-1-r) / 2 m)},
$$

where $F$ is an arbitrary domain in $T^{N}$ such that $\bar{F} \cap K=\emptyset, 2 \leq q \leq \infty$.

For any number $\tau \geq 0$ introduce the following functions (kernels):

$$
D_{\tau, \lambda}^{s}(x)=(2 \pi)^{-N / 2} \sum_{\Lambda(\lambda)} P_{m}^{\tau}(n)\left(1-\frac{P_{m}(n)}{\lambda}\right)^{s} \exp i n x
$$

Note that $D_{0, \lambda}^{s}(x)=D_{\lambda}^{s}(x)$.

Lemma 3.6. Let $\left|x^{\prime}\right|>\varepsilon$, where $\varepsilon>0$ is an arbitrary small number, and let s satisfy (2.8). Then for any nonnegative number $\tau$ the following relation is true.

$$
D_{\tau, \lambda}^{s}(x)-\lambda^{\tau} D_{\lambda}^{S}(x)=O(1) \lambda^{(N-s-2-(r / 2)-((N-1-r) / 2 m) / 2(m+1))+\tau} .
$$

Proof. If $\tau$ is an integer, then (3.20) follows directly from Lemma 3.3 and the relation

$$
\lambda^{-1} D_{k+1, \lambda}^{s}(x)=D_{k, \lambda}^{s}(x)-D_{k, \lambda}^{s+1}(x)
$$

If $\tau$ is not an integer, then write $\tau=k+\delta$, where $k$ is an integer and $\delta \in(0,1)$. Then there is a positive function $\rho(t)$, such that $\rho \leq$ const $t^{\delta-1}$ and

$$
\lambda^{-\delta} D_{k+1, \lambda}^{s}(x)=D_{k, \lambda}^{s}(x)-\delta D_{k, \lambda}^{s+1}(x)+\int_{0}^{1} D_{k, t \lambda}^{s+1}(x) \rho(t) d t .
$$

Then statement of the Lemma 3.6 follows from the relation (3.22) and Lemma 3.3. Lemma 3.6 is proved. 
From Lemmas 3.5 and 3.6 we obtain the following.

Lemma 3.7. Let $K \subset \subset T^{N}$ be an arbitrary compact set, s satisfy (2.8), and $2 \leq q \leq \infty$. Then uniformly by $x \in K$ the following estimation is valid:

$$
\left\|D_{\tau, \lambda}^{s}(x-y)\right\|_{L_{q}(F)}=O\left(\lambda^{(N-s-2-(r / 2)-((N-1-r) / 2 m) / 2(m+1))+\tau}\right),
$$

where $F$ is an arbitrary domain in $T^{N}$ such that $\bar{F} \cap K=\emptyset$.

\section{Proof of the Main Result}

Let a distribution $f$ have a compact support and belong to the space $L_{p}^{-\alpha}\left(T^{N}\right)$, where $1<p \leq$ $2, \alpha>0$. Let $K$ be an arbitrary compact set from $T^{N} \backslash \operatorname{supp} f$ and $s$ satisfy (2.8). Then from (3.1) it follows that

$$
\left|E_{\lambda}^{s} f(x)\right| \leq\|f\|_{-\alpha, p}\left\|D_{\lambda}^{s}(x-y)\right\|_{\alpha, q, F^{\prime}}
$$

where $\|\cdot\|_{-\alpha, p}$ means a norm in the space $L_{p}^{-\alpha}\left(T^{N}\right)$ and $\|\cdot\|_{\alpha, q, F}$ means a norm in the space $L_{q}^{\alpha}(F), 1 / q=1-(1 / p)$, and supp $f \subset F$ such that $\bar{F} \cap K=\emptyset$.

Let $\tau=\alpha / 2(m+1)$. Then from inequality

$$
\left\|D_{\lambda}^{s}\right\|_{\alpha, q} \leq c\left\|D_{\tau, \lambda}^{s}\right\|_{L_{q}}
$$

and Lemma 3.7 it follows that

$$
E_{\lambda}^{S} f(x)=O(1)\|f\|_{-\alpha, p}
$$

uniformly by $x$ from $K$.

Statement of Theorem 2.1 follows from inequality (4.3).

\section{Acknowledgment}

This paper has been supported by Universiti Putra Malaysia under Research University Grant (RUGS) no. 05-01-11-1273RU.

\section{References}

[1] Sh. A. Alimov, V. A. Il'in, and E. M. Nikishin, "Convergence problems of multiple trigonometric series and spectral decompositions. I.," Russian Mathematical Surveys, vol. 31, no. 6, pp. 29-86, 1976.

[2] Sh. A. Alimov, V. A. Il'in, and E. M. Nikishin, "Problems of convergence of multiple trigonometric series and spectral decompositions II.," Russian Mathematical Surveys, vol. 32, no. 1, pp. 115-139, 1977.

[3] M. A. Pinsky, "Fourier inversion for piecewise smooth functions in several variables," Proceedings of the American Mathematical Society, vol. 118, no. 3, pp. 903-910, 1993.

[4] M. A. Pinsky and M. E. Taylor, "Pointwise Fourier inversion: a wave equation approach," The Journal of Fourier Analysis and Applications, vol. 3, no. 6, pp. 647-703, 1997. 
[5] M. Taylor, "Eigenfunction expansions and the Pinsky phenomenon on compact manifolds," The Journal of Fourier Analysis and Applications, vol. 7, no. 5, pp. 507-522, 2001.

[6] Sh. A. Alimov, "Spectral expansions of distributions," Doclady Math, vol. 331, no. 6, pp. 661-662, 1993.

[7] Sh. A. Alimov and A. A. Rakhimov, "On the localization of spectral expansions of distributions in a closed domain," Journal of Differential Equations, vol. 33, no. 1, pp. 80-82, 1997.

[8] Sh. A. Alimov and A. A. Rakhimov, "On the localization of spectral expansions of distributions," Journal of Differential Equations, vol. 32, no. 6, pp. 792-802, 1996.

[9] A. A. Rakhimov, "On the summability of multiple Fourier trigonometric series of distributions," Doclades of Russian Academy of Sciences, vol. 374, no. 1, pp. 20-22, 2000.

[10] A. A. Rakhimov, "Spectral expansions of distributions from negative Sobolev classes," Journal of Differential Equations, vol. 32, no. 7, pp. 1000-1013, 1996.

[11] A. A. Rakhimov, "Localization conditions of spectral expansions of distributions connected with Laplace operator on sphere," Acta NUUz, vol. 2, pp. 47-49, 2006.

[12] A. A. Rakhimov, "On the localization conditions of the Reiszean means of distribution expansions of the Fourier series by eigenfunctions of the Laplace operator on sphere," Journal Izvestiya Vuzov, vol. 3-4, pp. 47-50, 2003.

[13] A. A. Rakhimov, "On the localization of spectral expansions of distributions with compact support connected with Schrodinger's operator," Journal of Astrophysics and Applied Mathematics, vol. 1, pp. 21-32, 2010.

[14] H. Triebel, Interpolation Theory, Function Spaces, Differential Operators, vol. 18 of North-Holland Mathematical Library, North-Holland Publishing, Amsterdam, The Netherlands, 1978.

[15] L. Hormander, "The spectral function of an elliptic operator," Acta Mathematica, vol. 121, no. 3-4, pp. 193-218, 1968.

[16] E. M. Stein and G. Weiss, Introduction to Fourier analysis on Euclidean spaces, Princeton University Press, Princeton, NJ, USA, 1971. 


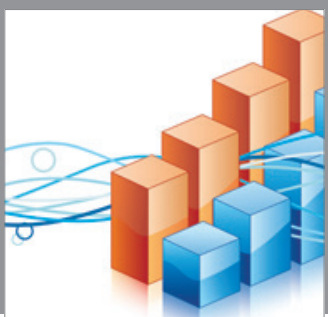

Advances in

Operations Research

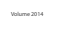

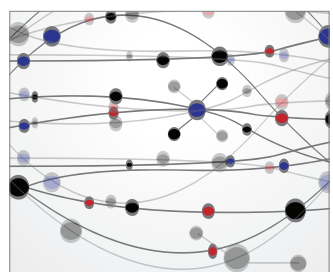

\section{The Scientific} World Journal
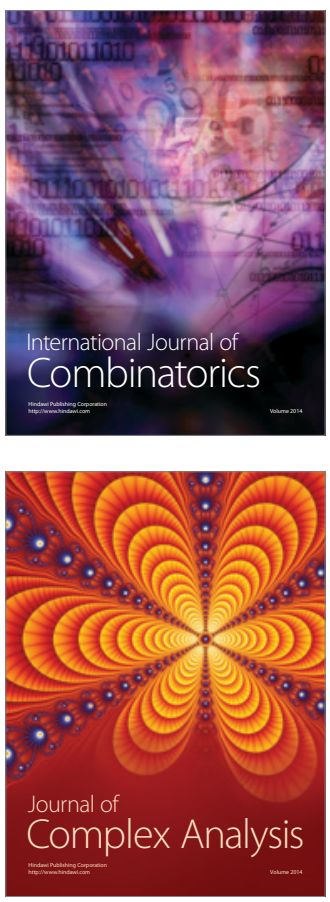

International Journal of

Mathematics and

Mathematical

Sciences
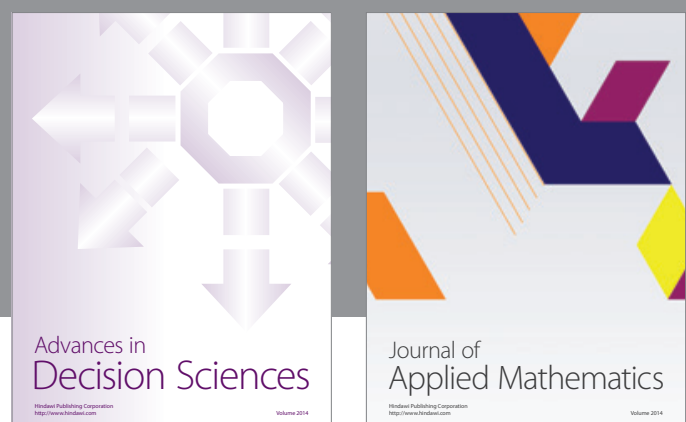

Journal of

Applied Mathematics
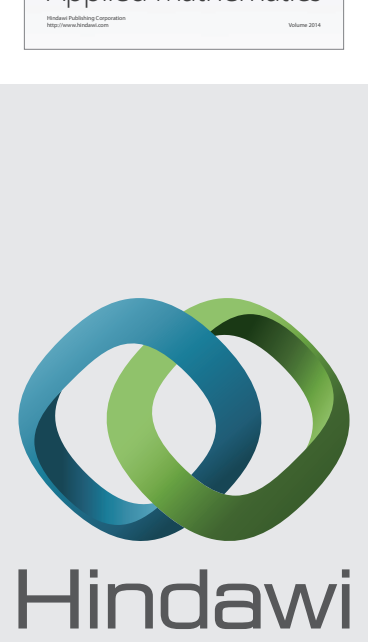

Submit your manuscripts at http://www.hindawi.com
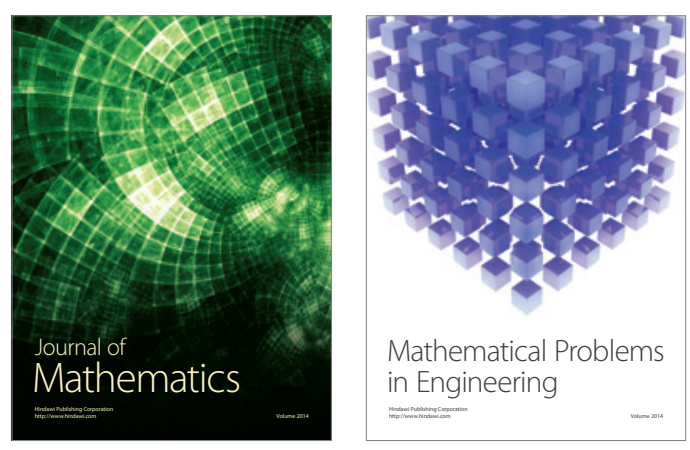

Mathematical Problems in Engineering
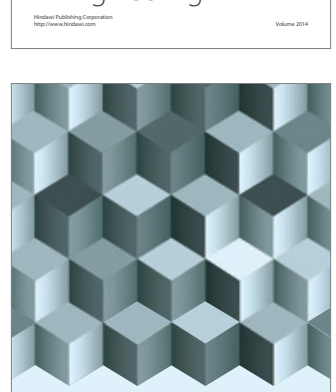

Journal of

Function Spaces
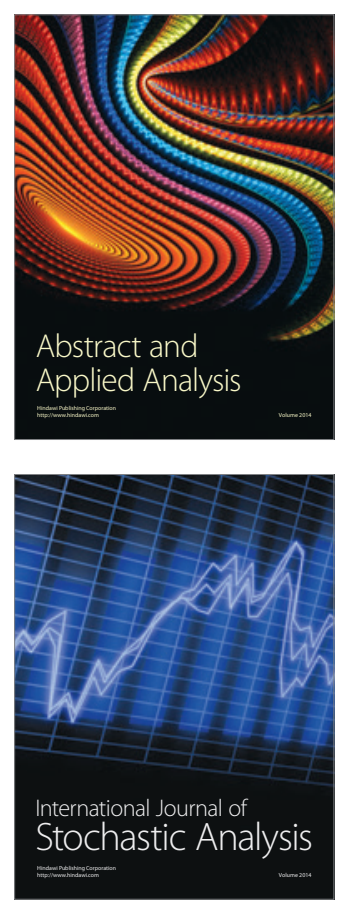

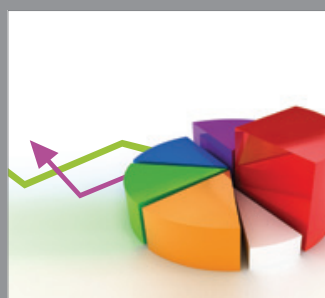

ournal of

Probability and Statistics

Promensencen
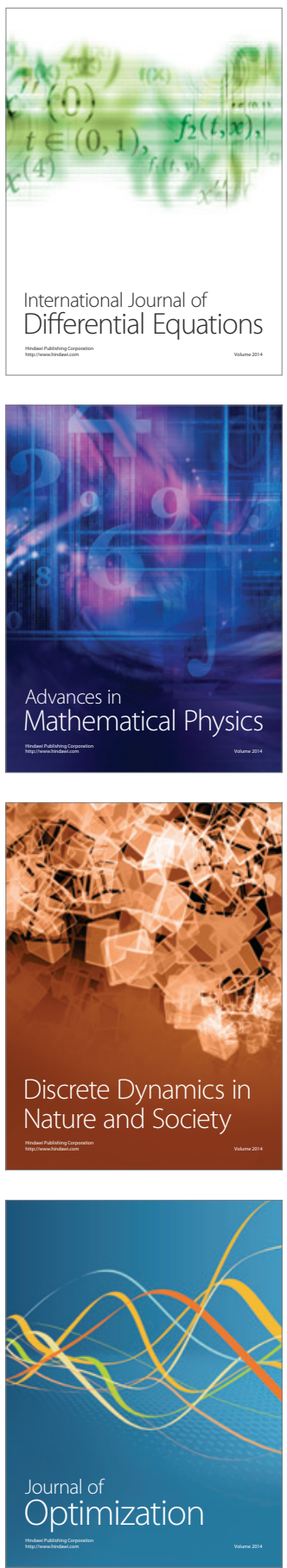